\title{
PKC $\zeta$, MMP-2 and MMP-9 expression in lung adenocarcinoma and association with a metastatic phenotype
}

\author{
XIAOSHAN CAI ${ }^{1}$, HONGGUANG ZHU ${ }^{2}$ and YING LI ${ }^{1}$ \\ ${ }^{1}$ Department of Pathology, Second People's Hospital of Weifang, Weifang, Shandong 261041; \\ ${ }^{2}$ Department of Dentistry, Weifang People's Hospital, Weifang, Shandong 261000, P.R. China
}

Received January 19, 2017; Accepted August 1, 2017

DOI: $10.3892 / \mathrm{mmr} .2017 .7634$

\begin{abstract}
The aim of the present study was to investigate protein kinase $\mathrm{C} \zeta$ type (PKC $\zeta$ ), matrix metalloproteinase (MMP)-2 and MMP-9 expression in lung adenocarcinoma and to define their association with in vitro invasion and metastatic capacity. PKC $\zeta$, MMP-2 and MMP-9 expression was assessed by immunohistochemistry in 110 cases of lung adenocarcinoma. PKC $\zeta$ small interfering (si)RNA was transfected into A549 cells, and western blotting was used to confirm PKC $\zeta$-knockdown in transfected cells and to measure MMP-2 and MMP-9 levels. A Transwell invasion assay was used to detect in vitro invasive capacity. The rates of positive PKC $\zeta$, MMP-2 and MMP-9 staining in lung adenocarcinoma tissues were 52.73, 55.45 and $61.82 \%$, respectively. $\mathrm{PKC} \zeta$ expression was increased in malignant tissues compared with adjacent normal lung tissues and was associated with lymph node metastasis $(\mathrm{P}<0.05)$, although it was not associated with any other clinicopathological parameters, including sex, age, tumor size, smoking status or distant metastases (all P>0.05). PKC $\zeta$, MMP-2 and MMP-9 expression was markedly decreased in siPKC $\zeta$-treated A549 cells, which exhibited a significantly decreased invasive capacity in the Transwell invasion assay $(\mathrm{P}<0.05)$. In conclusion, $\mathrm{PKC} \zeta$ promoted lung adenocarcinoma invasion and metastasis, and its expression was associated with MMP-2 and MMP-9 expression. PKC $\zeta$ may be a potential target for gene therapy in lung adenocarcinoma.
\end{abstract}

\section{Introduction}

Lung cancer is one of the commonest malignant carcinomas in the world, and its incidence is increasing in a number of countries. Worldwide, lung cancer is the leading cause of

Correspondence to: Dr Hongguang Zhu, Department of Dentistry, Weifang People's Hospital, 151 Guangwen Street, Weifang, Shandong 261000, P.R. China

E-mail: hongguangzhuwf@126.com

Key words: lung adenocarcinoma, protein kinase C $\zeta$ type, matrix metalloproteinase-2, matrix metalloproteinase-9, invasion, metastasis death from malignant tumor, accounting for $\sim 30 \%$ of all cancer-associated mortality (1). Lung adenocarcinoma is the principal subtype of lung cancer, and metastasis is the leading cause of mortality in patients with lung adenocarcinoma.

The protein kinase C (PKC) family regulates cell growth, differentiation, metabolism and transcriptional activation. PKCs may affect the invasion and metastasis of tumor cells. PKC $\zeta$ is a member of the PKC family that serves important roles in cell growth, metabolism and other associated signal transduction pathways $(2,3)$. It has been established that PKC $\zeta$ is a tumor suppressor for numerous types of human cancer (4). However, studies have additionally identified pro-oncogenic functions of $\mathrm{PKC} \zeta$, although a complete understanding of the detailed molecular mechanisms is lacking (2-4). Additionally, it has been suggested that $\mathrm{PKC} \zeta$ may be involved in inflammatory responses to diverse stimuli in vitro and in vivo (5-8). However, $\mathrm{PKC} \zeta$ expression in lung adenocarcinoma and the possible outcomes of PKC $\zeta$ signaling in the context of lung adenocarcinoma remain to be completely elucidated.

Matrix metalloproteinases (MMPs) are able to degrade the extracellular matrix and basement membrane, and serve important roles in promoting tumor invasion and metastasis $(9,10)$. MMPs proteolytically activate or degrade a variety of non-matrix substrates, including cytokines and chemokines, exerting a regulatory function in inflammation and immunity (11). At present, the most well-established roles for MMPs are in colorectal carcinogenesis, wherein MMP-2 and MMP-9 have been implicated in colon cancer progression and metastasis (12). Studies into the role of metalloproteinases and their inhibitors in lung adenocarcinoma are limited, and the results have been varied $(13,14)$.

Recently, studies have demonstrated that PKCs may promote the metastasis of tumor cells in breast cancer, glioma and other malignancies $(15,16)$. PKC $\zeta$ is able to activate the mitogen-activated protein kinase (MAPK) signaling pathway, which terminates with extracellular signal-regulated kinase (ERK) phosphorylation and consequent promotion of MMP-2 and MMP-9 secretion, which may facilitate invasion and metastasis $(17,18)$. However, there have been few studies focusing on lung adenocarcinoma, and whether PKC $\zeta$ may mediate the invasion and metastasis of lung adenocarcinoma by regulating MMP-2 and MMP-9 secretion remains unknown.

In the present study, $\mathrm{PKC} \zeta, \mathrm{MMP}-2$ and MMP-9 expression was assessed in lung adenocarcinoma and adjacent normal 
lung tissues using immunohistochemistry, and associations between their relative expression levels were analyzed. PKC $\zeta$ was knocked down in the lung adenocarcinoma cell line A549, and invasive capacity, and MMP-2 and MMP-9 expression were observed, in order to examine the effects of PKC $\zeta$ on invasion and metastasis in lung adenocarcinoma and to provide a novel method for the treatment of lung adenocarcinoma.

\section{Materials and methods}

Specimen collection. The present study included 110 patients with invasive lung adenocarcinoma (including all subtypes) who underwent histological diagnosis at the Second People's Hospital of Weifang (Weifang, China) between January 2012 and December 2014. Cases with preoperative therapy or a history of other known malignancies were excluded. Medical records were reviewed for clinicopathological features, including sex, age, tumor size, smoking status, lymph node metastasis, distant metastasis and pathological tumor, node, metastasis (pTNM) stage. Patients were divided into two groups by age ( $\leq 60$ years and $>60$ years) and smoking status [smokers ( $>5$ pack-year history) and non-smokers]. The pTNM stage was evaluated in accordance with the 7th lung cancer TNM classification and staging system (19). Adjacent normal lung tissue (taken $5 \mathrm{~cm}$ from the edge of the cancerous tissue) was used as the control.

Among the 110 lung adenocarcinoma patients: 59 were male and 51 were female; 66 were $\leq 60$ years old and 44 were $>60$; and 44 were smokers and 66 were non-smokers. Regarding pTNM stage, 36 were stages I+II and 74 were stages III+IV. The present study was approved by the Institutional Ethics Committee of Second People's Hospital of Weifang, and written informed consent was obtained from all participants.

Reagents. Anti-PKC' (TA312044), anti-MMP-2 (TA806846) and anti-MMP-9 (TA353338) antibodies were purchased from OriGene Technologies, Inc. (Beijing, China). Cell culture plates, Matrigel and Transwell chambers were purchased from Sigma-Aldrich (Merck KGaA, Darmstadt, Germany). Lipofectamine 2000 transfection reagent was purchased from Invitrogen (Thermo Fisher Scientific, Inc., Waltham, MA, USA) and RIPA lysis buffer was purchased from Beyotime Institute of Biotechnology (Haimen, China). The A549 cell line was purchased from the American Type Culture Collection (Manassas, VA, USA).

Immunohistochemistry. Immunohistochemical staining was performed on 4- $\mu \mathrm{m}$, formalin-fixed, paraffin-embedded sections. PKC $\zeta$ primary antibody was diluted 1:200 and manually applied to sections. All steps were performed in accordance with the manufacturer's protocol. MMP-2 and MMP-9 were not diluted for these experiments. PBS was used as the negative control. Staining intensity and the percentage of positive cells were evaluated under a microscope (BX53; Olympus Corporation, Tokyo, Japan) in 5 high-magnification fields of vision, and 100 cells were counted in each field. The specific methods were performed according to a previous study (18).

Cell culture. The lung adenocarcinoma cell line A549 was cultured in F12K culture medium (21127-022; Invitrogen;
Thermo Fisher Scientific, Inc.) containing 10\% fetal bovine serum (Gibco, Thermo Fisher Scientific, Inc.) at $37^{\circ} \mathrm{C}$ in $5 \% \mathrm{CO}_{2}$. Experiments were performed on cells in the logarithmic growth phase. The cells were divided into 3 groups as follows: Control group, A549 cells without any treatment; Scr/A549 group, A549 cells transiently transfected with empty plasmid; and small interfering (si)PKC $\zeta / A 549$ group, A549 cells transiently transfected with the PKC $\zeta$ target fragment 5'-GAGGAAGTGAGAGACATGTGT-3'. A total of $0.4 \mu \mathrm{g}$ plasmid/siRNA were transfected into the Scr/A549 group and the (si)PKC /A549 group. All the vectors were synthesized by Shanghai GeneChem Co., Ltd. (Shanghai, China). Transfections were performed using Lipofectamine 2000 (Invitrogen; Thermo Fisher Scientific, Inc.) according to the manufacturer's protocol. The subsequent experimentation commenced $48 \mathrm{~h}$ following transfection.

Western blotting. For Western blot analysis, cells or tissues were directly lysed in RIPA lysis buffer. Aliquots of $50 \mu \mathrm{g}$ protein were separated by $10 \%$ SDS-PAGE and transferred onto polyvinylidene difluoride membranes (EMD Millipore, Billerica, MA, USA). The membranes were blocked in 5\% skimmed milk for $1 \mathrm{~h}$ at room temperature, and then immunoblotted using the appropriate primary antibodies at $4^{\circ} \mathrm{C}$ overnight and the HRP conjugated secondary antibodies at $37^{\circ} \mathrm{C}$ for $2 \mathrm{~h}$. They were visualized by using enhanced chemiluminescence reagents ECL (Pierce; Thermo Fisher Scientific, Inc.). Western blot data in the present study are representative from three independent experiments. The intensities of bands in western blots were quantified by densitometry analysis using AlphaImager HP (version 3.4.0; ProteinSimple, San Leandro, CA, USA) and NIH ImageJ software (version 1.44; National Institutes of Health, Bethesda, MD, USA). The following commercial antibodies were used in this study: PKC $\zeta$ (TA312044; 1:1,000), MMP-2 (TA806846; 1:1,000) and MMP-9 (TA353338; 1:1,000) (all from OriGene Technologies, Inc.), $\beta$-actin $(4970 ; 1: 1,000)$ and HRP-linked anti-rabbit IgG antibody (7074; 1:2,000) (both from Cell Signaling Technology, Inc., Danvers, MA, USA).

Transwell invasion assay. Matrigel was added to the top chamber of a Transwell system to form the matrix layer. To this matrix was added $100 \mu 1\left(1 \times 10^{5}\right) \mathrm{Scr} / \mathrm{A} 549$ or siPKC $\zeta / A 549$ cells; epidermal growth factor was added into the lower chamber $(500 \mu \mathrm{l} /$ well). The Transwell device was placed in an incubator $\left(37^{\circ} \mathrm{C} ; 5 \% \mathrm{CO}_{2}\right)$ for $24 \mathrm{~h}$. Following incubation, invaded cells were fixed for $1 \mathrm{~min}$ in precooled methanol and Giemsa stained for $30 \mathrm{~min}$ at room temperature. All experiments were repeated at least three times. The number of invading cells was counted under a microscope (IX71; Olympus Corporation) in five predetermined fields, total magnification, x200, using CellSens Standard (version 1.7; Olympus Corporation).

Statistical analysis. All statistical analyses were performed using SPSS version 19.0 (IBM Corp., Armonk, NY, USA). Data are presented as the mean \pm standard deviation. Statistical significance was evaluated using Student's t-test or $\chi^{2}$ test. $\mathrm{P}<0.05$ was considered to indicate a statistically significant difference. 
A

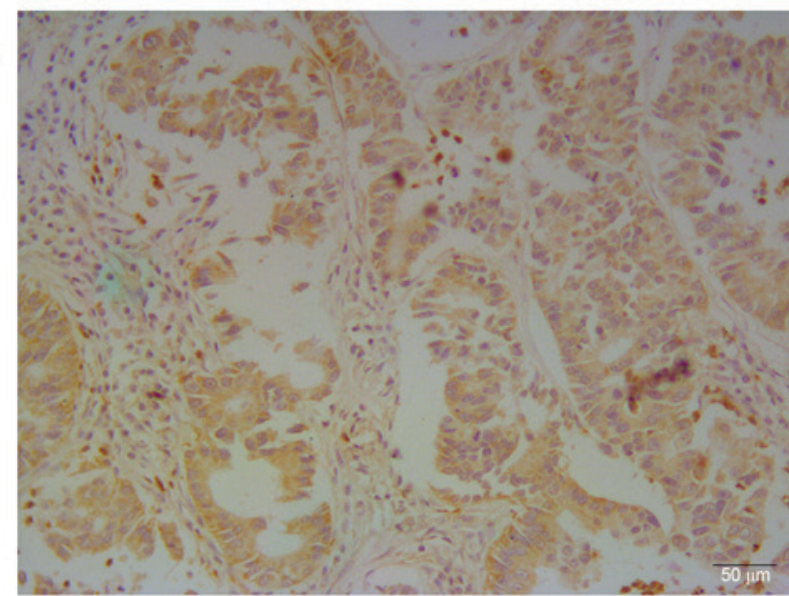

B

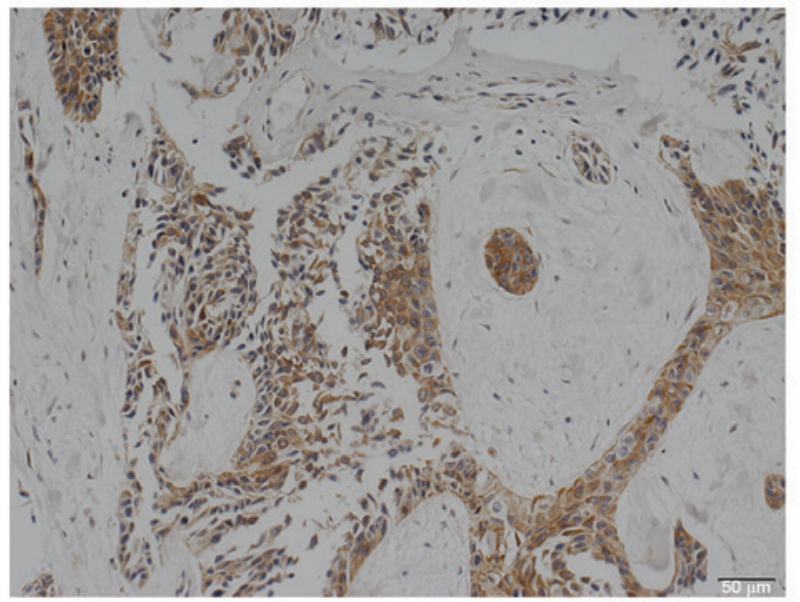

C

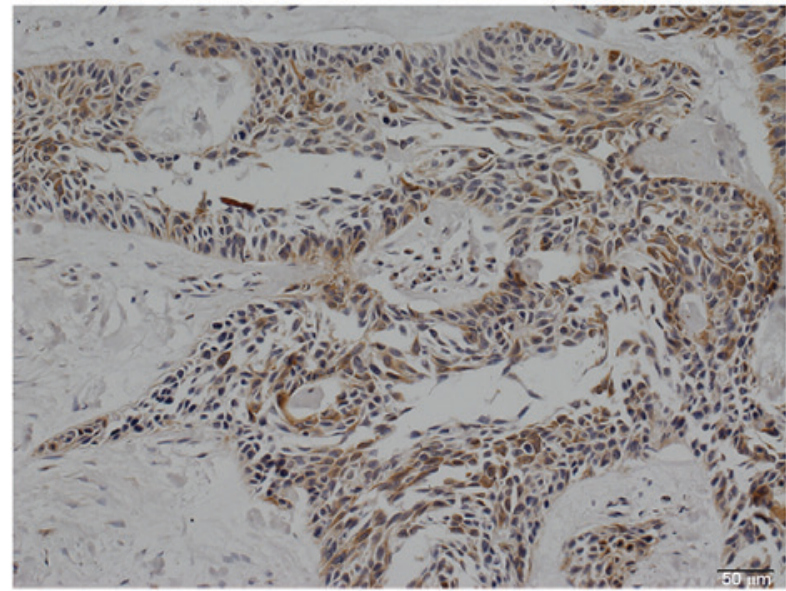

Figure 1. Expression of PKC $\zeta$, MMP-2 and MMP-9 in lung adenocarcinoma, analyzed using immunohistochemistry. (A) Positive PKC $\zeta$, (B) MMP-2 and (C) MMP-9 staining was observed in the cytoplasm of lung adenocarcinoma sections (magnification, $\mathrm{x} 100$ ). $\mathrm{PKC} \zeta$, protein kinase $\mathrm{C} \zeta$ type; MMP, matrix metalloproteinase.

\section{Results}

Immunohistochemical findings. PKC $\zeta$ was expressed in the cytoplasm of lung adenocarcinoma cells (Fig. 1A). Positive PKC $\zeta$ staining was detected in 58 (52.73\%) lung adenocarcinoma samples, while only 5 (4.50\%) normal lung tissues exhibited weak positive staining. The difference was statistically significant $\left(\chi^{2}=62.479 ; \mathrm{P}<0.01\right)$. The rate of positive $\mathrm{PKC} \zeta$ staining in lung adenocarcinomas with lymph
Table I. Expression of PKC $\zeta$ in lung adenocarcinoma and association with clinical pathological indices.

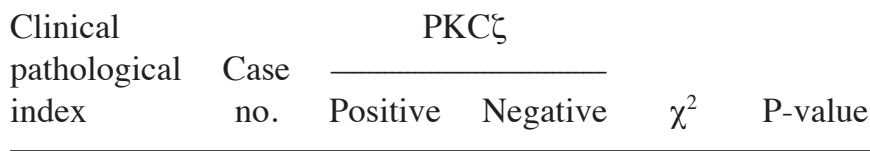

Gender

$\begin{array}{llllll}\text { Male } & 59 & 32 & 27 & 0.116 & 0.848 \\ \text { Female } & 51 & 26 & 25 & & \end{array}$

Age, years

$\begin{array}{llllll}\leq 60 & 66 & 30 & 36 & 3.501 & 0.08 \\ >60 & 44 & 28 & 16 & & \end{array}$

Diameter of

tumor, $\mathrm{cm}$

$\begin{array}{llllll}\leq 3 & 40 & 17 & 23 & 2.638 & 0.116 \\ >3 & 70 & 41 & 29 & & \\ \text { Smoker } & & & & & \\ \text { Yes } & 44 & 21 & 23 & 0.736 & 0.439 \\ \text { No } & 66 & 37 & 29 & & \end{array}$

Metastasis

of LN

$\begin{array}{clllll}\text { Yes } & 39 & 26 & 13 & 4.710 & 0.045 \\ \text { No } & 71 & 32 & 39 & & \end{array}$

Distant

metastasis

\begin{tabular}{llllll} 
Yes & 42 & 27 & 15 & 3.642 & 0.077 \\
No & 68 & 31 & 37 & & \\
TNM stage & & & & & \\
I+II & 36 & 16 & 20 & 1.473 & 0.309 \\
III+IV & 74 & 42 & 32 & & \\
\hline
\end{tabular}

LN, lymph node; TNM, tumor, node, metastasis; $\mathrm{PKC} \zeta$, protein kinase $\mathrm{C} \zeta$ type.

node metastases $(64.30 \%)$ was increased compared with non-metastatic samples $(45.60 \%)(\mathrm{P}=0.017)$. The differences among other clinicopathological parameters were not significant (Table I).

MMP-2 and MMP-9 were primarily expressed in the cytoplasm of lung adenocarcinomas (Fig. 1B and C); the rate of positive staining was 55.45 and $61.82 \%$, respectively. PKC $\zeta$ expression was associated with MMP-2 ( $\mathrm{P}=0.012)$ and MMP-9 $(\mathrm{P}=0.006)$ expression in lung adenocarcinoma (Table II).

Western blot analysis results. PKC $\zeta$ expression siPKC $\zeta / A 549$ cells was markedly decreased compared with Scr/A549 cells, confirming that the reagent successfully disrupted the expression of the target gene (Fig. 2). In addition, MMP-2 and MMP-9 protein expression in siPKC $\zeta / A 549$ cells was markedly decreased compared with Scr/A549 cells (Fig. 2).

Transwell invasion assay findings. Fewer siPKC /A549 cells invaded through the membrane and into the bottom chamber 
Table II. Association between PKC $\zeta$, MMP-2 and MMP-9 expression in lung adenocarcinoma.

\begin{tabular}{lcccccc}
\hline & & \multicolumn{2}{c}{ PKC 5} & & \\
Group & Case no. & Positive & Negative & $\chi^{2}$ & P-value \\
\hline MMP-2 & & & & & \\
Positive & 61 & 39 & 22 & 6.900 & 0.012 \\
Negative & 49 & 19 & 30 & & \\
MMP-9 & & & & & \\
Positive & 68 & 43 & 25 & 7.889 & 0.006 \\
Negative & 42 & 15 & 27 & & \\
\hline
\end{tabular}

PKC $\zeta$, protein kinase $\mathrm{C} \zeta$ type; MMP, matrix metalloproteinase.

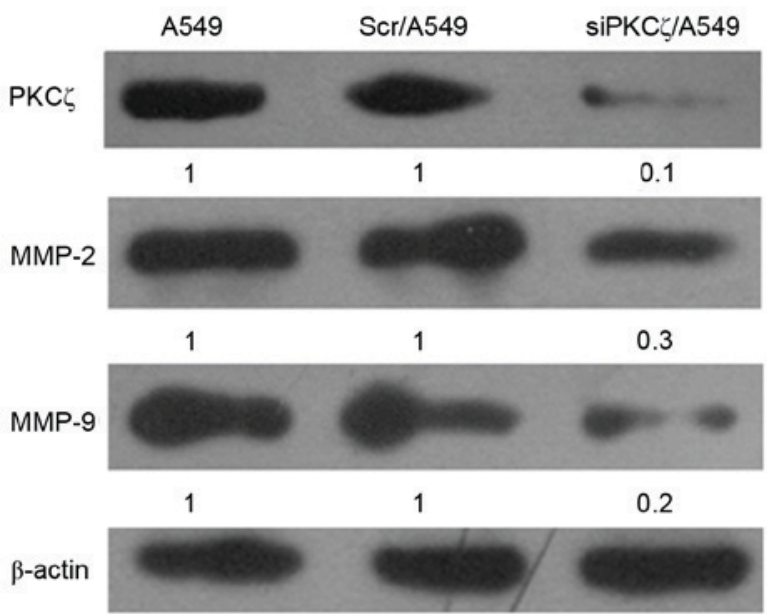

Figure 2. PKC $\zeta$ siRNA-transfected A549 cells. Following PKC $\zeta$ siRNA transfection, $\mathrm{PKC} \zeta, \mathrm{MMP}-2$ and MMP-9 were immunoprecipitated and proteins were analyzed using western blotting. $\mathrm{PKC} \zeta$, MMP-2 and MMP-9 expression was decreased in the siPKC $\zeta / A 549$ group. $\mathrm{PKC} \zeta$, protein kinase $\mathrm{C}$ $\zeta$ type; MMP, matrix metalloproteinase; siRNA, small interfering RNA; Scr, scramble.

compared with Scr/A549 cells $(\mathrm{P}<0.05)$, suggesting that $\mathrm{PKC} \zeta$ downregulation was able to decreased the invasive ability of lung adenocarcinoma cells (Fig. 3).

\section{Discussion}

In the present study, it was observed that positive $\mathrm{PKC} \xi$ expression in lung adenocarcinoma was associated with lymph node metastasis, and MMP-2 and MMP-9 expression. It was additionally observed that MMP-2 and MMP-9 expression was decreased in A549 cells following PKC $\zeta$ knockdown by siRNA, which weakened the invasive ability of the cells in vitro.

PKCs are lipid-dependent serine/threonine protein kinases present in mammalian cells that serve important roles in growth, metabolism, proliferation and cytoskeletal remodeling. PKCs are also important intracellular signaling molecules that have been demonstrated to act as oncogenes and tumor suppressors, depending on the cellular context and upon which

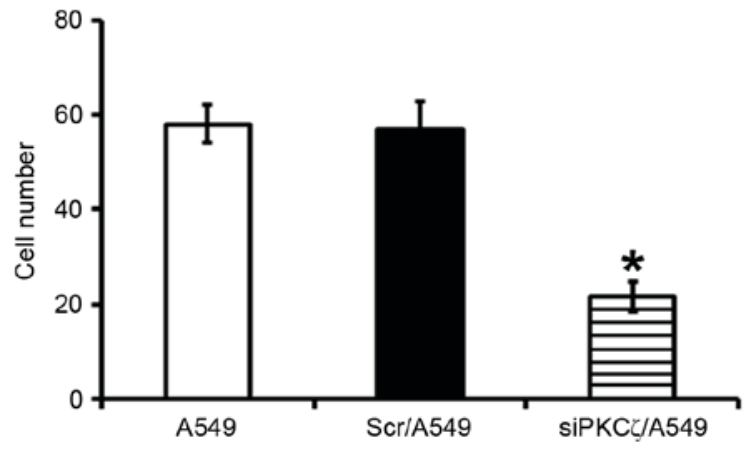

Figure 3. In vitro invasive ability of A549 cells with and without PKC $\zeta$. In vitro invasion was detected using a Transwell invasion assay, which demonstrated significantly decreased levels of invasion in the siPKC $\zeta / A 549$ group compared with Scr/A549 cells. ${ }^{*} \mathrm{P}<0.05$ vs. Scr/A549. PKC $\zeta$, protein kinase $\mathrm{C} \zeta$ type; siRNA, small interfering RNA; Scr, scramble.

protein adaptors interact with which PKC isoforms (20-23). PKC isozymes comprise three classes: Conventional (cPKC, $\alpha$, $\beta$ and $\gamma$ ), novel (nPKC, $\delta, \varepsilon, \eta$ and $\theta$ ) and atypical (aPKC, $\zeta$ and ı). Different PKC isotypes are known to serve distinct regulatory roles. $\mathrm{PKC} \zeta$ is an important subtype of atypical PKCs that is involved in numerous signal transduction pathways.

PKC family proteins have been intensively studied due to their association with cancer. Previous studies have demonstrated that $\mathrm{PKC} \zeta$ may promote tumor cell chemotaxis in glioma, liver cancer and breast cancer, thus promoting cancer cell invasion and metastasis $(16,24)$. When PKC $\zeta$ is activated, it may phosphorylate Lim domain kinase 1 and cofilin, promoting F-actin depolymerization and polymerization, respectively, which affects the cytoskeleton structure and inhibits cancer cell chemotaxis and migration. Moreover, PKC $\zeta$ is able to activate integrin- $\beta 1$, which enhances adhesion between cells, activates the MAPK pathway, and promotes vascular endothelial growth factor (VEGF) expression and angiogenesis, which may consequently promote tumor invasion and metastasis $(17,18)$.

Ma et al (25) observed that $\mathrm{PKC} \zeta$ is involved in lung cancer cell adhesion and chemotaxis, and thus may affect the invasion and metastasis of lung cancer. In the present study, it was demonstrated that the rate of positive $\mathrm{PKC} \zeta$ staining in patient-derived lung adenocarcinoma paraffin sections assayed by immunohistochemistry was significantly increased compared with adjacent tissues, and that $\mathrm{PKC} \zeta$ expression was associated with lymph node metastasis. This result also suggested that PKC $\zeta$ affected the invasion and metastasis of lung adenocarcinoma. In vitro Transwell invasion experiments using A549 lung adenocarcinoma cells further confirmed that reducing PKC $\zeta$ inhibited the invasive capacity of tumor cells. Therefore, the results of the present study demonstrated that PKC $\zeta$ was able to promote the invasion and metastasis of lung adenocarcinoma through in vitro and in vivo methods.

MMPs are a family of $\mathrm{Zn}^{2+}$-dependent endopeptidases that are able to degrade the extracellular matrix and basement membrane, and serve an important role in physiological and pathological processes. They have been regarded as critical factors that promote tumor cell invasion. MMP-2 and MMP-9 are the most important enzymes for type IV collagen degradation, and serve important roles in tumor 
angiogenesis, invasion and metastasis $(26,27)$. The mechanism underlying this effect involves the increase of VEGF secretion from tumor cells induced by MMP-2 and MMP-9, promoting invasion and metastasis, which is dependent on MAPK activation and ERK phosphorylation. In addition, MMP-9 expression is known to cause emphysema in chronic obstructive pulmonary disorder and angiogenesis/metastasis in lung cancer (28).

Studies have demonstrated that MMP-2 and MMP-9 expression in non-small cell lung cancer is significantly increased compared with normal tissue adjacent to the cancer, and that their expression levels are associated with pathological grading and staging, invasion and metastasis $(20,29)$. PKC $\zeta$ was able to activate MAPK and the MAPK signaling pathway, and promote VEGF overexpression, angiogenesis, tumor invasion and metastasis (17). In the present study, it was observed that PKC $\zeta$ expression was associated with the expression of MMP-2 and MMP-9 in lung adenocarcinoma, using immunohistochemical detection. By decreasing the expression of PKC $\zeta$ in A549 cells, the invasiveness of siPKC $\zeta / A 549$ cells decreased significantly; decreased $\mathrm{PKC} \zeta$ expression coincided with reduced secretion of MMP-2 and MMP-9. The above results suggested that the $\mathrm{PKC} \zeta$ may promote lung cancer invasion and metastasis by affecting MMP-2 and MMP-9 secretion in lung adenocarcinoma cells.

In conclusion, $\mathrm{PKC} \zeta$ expression was associated with the invasion and metastasis of lung adenocarcinoma, making PKC $\zeta$ a potential target for gene therapy in lung cancer and providing a theoretical basis for enhancing the survival rate of patients with lung adenocarcinoma. PKC $\zeta$, MMP-2 and MMP-9 synergistically promoted lung cancer invasion and metastasis, although the specific mechanism remains unclear and requires further research.

\section{Acknowledgements}

The present study was supported by the Program of Weifang Health Bureau in China (grant no. 2012012) and the Program of Bureau of Science and Technology in Weifang Kuiwen District in China (grant no. 201620).

\section{References}

1. Lu J, Wang W, Xu M, Li Y, Chen C and Wang X: A global view of regulatory networks in lung cancer: An approach to understand homogeneity and heterogeneity. Semin Cancer Biol 42: 31-38, 2017

2. Wu J, Zhang B, Wu M, Li H, Niu R, Ying G and Zhang N: Screening of a PKC zeta-specific kinase inhibitor PKCzI257.3 which inhibits EGF-induced breast cancer cell chemotaxis Invest New Drugs 28: 268-275, 2010.

3. Butler AM, Scotti BML, Li S, Smith KE, Fields AP and Murray NR: Protein kinase $\mathrm{C}$ zeta regulates human pancreatic cancer cell transformed growth and invasion through a STAT3-dependent mechanism. PLoS One 8: e72061, 2013.

4. Queisser MA, Dada LA, Deiss-Yehiely N, Angulo M, Zhou G, Kouri FM, Knab LM, Liu J, Stegh AH, DeCamp MM, et al: HOIL-1L functions as the PKC $\zeta$ ubiquitin ligase to promote lung tumor growth. Am J Respir Crit Care Med 190: 688-698, 2014.

5. LaVallie ER, Chockalingam PS, Collins-Racie LA, Freeman BA, Keohan CC, Leitges M, Dorner AJ, Morris EA, Majumdar MK and Arai M: Protein kinase Czeta is up-regulated in osteoarthritic cartilage and is required for activation of NF-kappaB by tumor necrosis factor and interleukin-1 in articular chondrocytes. J Biol Chem 281: 24124-24137, 2006.
6. Abdel-Halim M, Darwish SS, ElHady AK, Hoppstädter J, Abadi AH, Hartmann RW, Kiemer AK and Engel M: Pharmacological inhibition of protein kinase $\mathrm{C}(\mathrm{PKC}) \zeta$ downregulates the expression of cytokines involved in the pathogenesis of chronic obstructive pulmonary disease (COPD). Eur J Pharm Sci 93: 405-409, 2016

7. Diaz-Meco MT and Moscat J: The atypical PKCs in inflammation: NF-кB and beyond. Immunol Rev 246: 154-167, 2012.

8. Morin C, Fortin S and Rousseau E: Bronchial inflammation induced $\mathrm{PKC} \zeta$ over-expression: Involvement in mechanical properties of airway smooth muscle. Can J Physiol Pharmacol 90: 261-269, 2012.

9. Xu M, Wang HF and Zhang HZ: Expression of RECK and MMPs in hepatoblastoma and neuroblastoma and comparative analysis on the tumor metastasis. Asian Pac J Cancer Prev 16: 4007-4011, 2015.

10. Merdad A, Karim S, Schulten HJ, Dallol A, Buhmeida A, Al-Thubaity F, Gari MA, Chaudhary AG, Abuzenadah AM and Al-Qahtani MH: Expression of matrix metalloproteinases (MMPs) in primary human breast cancer: MMP-9 as a potential biomarker for cancer invasion and metastasis. Anticancer Res 34: 1355-1366, 2014.

11. Parks WC, Wilson CL and López-Boado YS: Matrix metalloproteinases as modulators of inflammation and innate immunity. Nat Rev Immunol 4: 617-629, 2004.

12. Babykutty S, Suboj P, Srinivas P, Nair AS, Chandramohan K and Gopala $S$ : Insidious role of nitric oxide in migration/invasion of colon cancer cells by upregulating MMP-2/9 via activation of cGMP-PKG-ERK signaling pathways. Clin Exp Metastasis 29: 471-492, 2012.

13. Kim HIe, Lee HS, Kim TH, Lee JS, Lee ST and Lee SJ: Growth-stimulatory activity of TIMP-2 is mediated through c-Src activation followed by activation of FAK, PI3-kinase/AKT, and ERK1/2 independent of MMP inhibition in lung adenocarcinoma cells. Oncotarget 6: 42905-42922, 2015.

14. Yamamura T, Nakanishi K, Hiroi S, Kumaki F, Sato H, Aida S and Kawai T: Expression of membrane-type-1-matrix metalloproteinase and metalloproteinase-2 in nonsmall cell lung carcinomas. Lung Cancer 35: 249-255, 2002.

15. Zhang F, Dong W, Zeng W, Zhang L, Zhang C, Qiu Y, Wang L, Yin X, Zhang C and Liang W: Naringenin prevents TGF- $\beta 1$ secretion from breast cancer and suppresses pulmonary metastasis by inhibiting PKC activation. Breast Cancer Res 18: 38, 2016.

16. Paul A, Danley M, Saha B, Tawfik O and Paul S: PKC $\zeta$ Promotes Breast Cancer Invasion by Regulating Expression of E-cadherin and Zonula Occludens-1 (ZO-1) via NFkB-p65. Sci Rep 5: 12520, 2015.

17. McCubrey JA, Steelman LS, Chappell WH, Abrams SL, Wong EW, Chang F, Lehmann B, Terrian DM, Milella M, Tafuri A, et al: Roles of the Raf/MEK/ERK pathway in cell growth, malignant transformation and drug resistance. Biochim Biophys Acta 1773: 1263-1284, 2007.

18. Yang S LH, Li W YL, Ni M ZL and Yin CaZB: Expression and correlation of PKC $\zeta$, MMP-2 and MMP-9 in breast cancer. Chin J Clin Exp Pathol 30: 958-962, 2014.

19. Mirsadraee S, Oswal D, Alizadeh Y, Caulo A and van Beek E Jr: The 7th lung cancer TNM classification and staging system: Review of the changes and implications. World J Radiol 4: 128-134, 2012

20. Rajagopalan S, Moyle MW, Joosten I and Long EO: DNA-PKcs controls an endosomal signaling pathway for a proinflammatory response by natural killer cells. Sci Signal 3: ra14, 2010.

21. Lim PS, Sutton CR and Rao S: Protein kinase C in the immune system: From signalling to chromatin regulation. Immunology 146: 508-522, 2015.

22. Fields AP and Regala RP: Protein kinase C iota: Human oncogene, prognostic marker and therapeutic target. Pharmacol Res 55: 487-497, 2007.

23. Antal CE, Hudson AM, Kang E, Zanca C, Wirth C, Stephenson NL, Trotter EW, Gallegos LL, Miller CJ, Furnari FB, et al: Cancer-associated protein kinase $\mathrm{C}$ mutations reveal kinase's role as tumor suppressor. Cell 160: 489-502, 2015.

24. Sun R, Gao P, Chen L, Ma D, Wang J, Oppenheim JJ and Zhang N: Protein kinase $\mathrm{C}$ zeta is required for epidermal growth factor-induced chemotaxis of human breast cancer cells. Cancer Res 65: 1433-1441, 2005.

25. Ma NZF, Guo HZX, Cao SNR and Zhang N: Correlation between $\mathrm{PKC} \zeta$ expression and invasion, metastasis and prognosis of adenoearcinoma of the lung. Chin J Clin Oncol 37: 557-560, 2010. 
26. Kuo HY, Huang YS, Tseng CH, Chen YC, Chang YW, Shih HM and $\mathrm{Wu} \mathrm{CW}$ : PML represses lung cancer metastasis by suppressing the nuclear EGFR-mediated transcriptional activation of MMP2. Cell Cycle 13: 3132-3142, 2014.

27. Yu Y, Ding Z, Jian H, Shen L, Zhu L and Lu S: Prognostic value of MMP9 activity level in resected stage I B lung adenocarcinoma. Cancer Med 5: 2323-2331, 2016.
28. King PT: Inflammation in chronic obstructive pulmonary disease and its role in cardiovascular disease and lung cancer. Clin Transl Med 4: 68, 2015

29. González-Arriaga P, Pascual T, García-Alvarez A, Fernández-Somoano A, López-Cima MF and Tardón A: Genetic polymorphisms in MMP 2, 9 and 3 genes modify lung cancer risk and survival. BMC Cancer 12: 121, 2012. 